\title{
COMUNICACIÓN
}

\section{Estudio comparativo de recuperación de formas parasitarias por tres diferentes métodos de enriquecimiento coproparasitológico}

\author{
GRACIELA T. NAVONE*, MARÍA I. GAMBOA*, LEONORA E. KOZUBSKY**, MARÍA E. COSTAS**, \\ MARÍA S. CARDOZO**, MIRIAM N. SISLIAUSKAS**, y MALENA GONZÁLEZ**
}

\section{COMPARATIVE STUDY OF PARASITIC FORMS RECUPERATION USING THREE DIFFERENT COPROPARASITOSCOPIC METHODS}

A total of 165 fecal samples were analyzed, using two sedimentation methods: Ritchie $(R)$, Carles Barthelemy $(C B)$, and one of flotation: Willis $(W)$, in order to optimize intestinal parasites diagnosis and to determine the techniques effectiveness. Parasites were found in $119(72,12 \%)$ of those analyzed. Significant differences were in protozoa recovery $(p<0,001)$, being observed $81.4 \%(R), 77.4 \%$ $(C B)$, and $57.8 \%(W)$. Blastocystis hominis, Giardia lamblia, Ascaris lumbricoides and Trichuris trichiura were recovered more frequency by means of sedimentation, being Ritchie's method the most effective one $(p<0.05)$.

Key words: intestinal parasites, sedimentation, flotation methods, serial fecal samples.

\section{INTRODUCCIÓN}

Las infecciones intestinales por helmintos y protozoos están entre las más comunes a nivel mundial, pues se distribuyen en todas las regiones tropicales y templadas del planeta; sin embargo, al ser más prevalentes en los países en desarrollo y en las comunidades más pobres, han sido consideradas como el resultado de las condiciones de vida y fueron subestimadas por los servicios de salud pública ${ }^{1,2}$. A pesar de esta situación, algunas parasitosis han cobrado importancia aún en países desarrollados (EEUU), asociadas con brotes de transmisión hídrica, tal es el caso de Cryptosporidium spp. y Giardia lamblia ${ }^{3,4}$.
En Argentina, las parasitosis intestinales constituyen un importante problema de salud pública, y son especialmente prevalentes en niños y adultos de poblaciones con necesidades básicas insatisfechas. Su distribución se encuentra influenciada por factores bióticos, abióticos y culturales $^{5-7}$.

Una de las maneras de diagnosticar las parasitosis de localización gastrointestinal y glándulas anexas, es mediante la aplicación de técnicas coproparasitológicas de enriquecimiento (de sedimentación y flotación), que permiten concentrar huevos, quistes y larvas en el menor volumen de materia fecal, determinar su presencia e identificarlos correctamente. La recuperación

\footnotetext{
* Centro de Estudios Parasitológicos y de Vectores (CEPAVE), CONICET-UNLP. Calle 2 n 584 La Plata (1900), Buenos Aires, Argentina. helmintos@ cepave.com.ar ** Cátedra de Parasitología, Ciencias Exactas, UNLP.
} 
de estas formas parasitarias plantea un problema de difícil solución a todo microscopista. Si bien es posible preparar frotis fecales a partir de heces frescas o material fecal conservado en SAF o formol, los métodos de concentración permiten que los quistes de protozoos y huevos de helmintos no pasen inadvertidos cuando están presentes en escaso número ${ }^{8}$.

Las técnicas de sedimentación, se utilizan para la observación de quistes de protozoos, huevos y larvas de helmintos, pero la desventaja de estas técnicas consiste en que los preparados contienen más residuos que los procesados por flotación. En este caso, el acetato de etilo se usa para extraer los residuos y las grasas de las heces y llevar los parásitos al fondo de la suspensión. Estas técnicas entonces son recomendadas por ser fáciles de realizar, tener baja probabilidad de errores técnicos y recuperar un amplio rango de organismos ${ }^{9}$.

Las técnicas de flotación permiten la separación de quistes de protozoos y huevos de ciertos helmintos del exceso de residuos mediante el uso de soluciones con elevada gravedad específica. Los elementos parasitarios son recuperados de la capa superficial y los residuos se mantienen en el fondo del tubo. Con estas técnicas los preparados son más limpios que los obtenidos por sedimentación. Sin embargo, algunos huevos (como los opérculados, o los densos como los estériles de Ascaris lumbricoides) no se concentran bien en las flotaciones; en estos casos se recomienda el uso de técnicas de sedimentación ${ }^{10}$. De todas maneras, en las flotaciones se observa también el fondo del tubo para asegurar la recuperación de todos los posibles organismos (9). Con el fin de maximizar la eficacia en la detección de parásitos intestinales se recomienda el uso de ambos métodos diagnósticos de manera conjunta ${ }^{9-12}$.

Las técnicas de sedimentación de Ritchie y Carles Barthelemy utilizan solución formolada al $10 \%$ y solución cítrica formolada respectivamente, además del acetato de etilo. La técnica de flotación de Willis usa solución saturada de cloruro de sodio $(\delta=1.200)$.

Durante el desarrollo de un trabajo ecoepidemiológico en un barrio periférico del partido de La Plata, en la provincia de Buenos, Argentina, se estudiaron los enteroparásitos mediante el uso de tres técnicas de enriquecimiento coproparasitológico, con el fin de optimizar el diagnóstico y la identificación de los parásitos hallados. Posteriormente, se compararon los resultados obtenidos, con el fin de determinar cuál de los tres métodos aplicados fue el más eficaz.El proposito de este trabajo es entregar estos resultados.

\section{MATERIAL Y MÉTODOS}

Se analizaron 165 muestras fecales seriadas formoladas, utilizando dos métodos de sedimentación: Ritchie (R) y Carles Barthelemy (CB) y uno de flotación: Willis (W).

\section{RESULTADOS Y DISCUSIÓN}

Se encontraron 119/ $165(72,12 \%)$ positivas a infección por algún parásito patógeno o potencialmente patógeno. En la tabla 1 y Figura 1 se presentan los resultados según la especie de párasito encontrada y según el grupos de parásitos. Se encontraron diferencias estadísticamente significativas en la recuperación de protozoos $(\mathrm{p}<0,001)$, observándose $81,4 \%(\mathrm{R})$, $77,4 \%$ (CB), y $57,8 \%(\mathrm{~W})$. Aunque para los helmintos las diferencias no fueron significativas, se recuperaron $77,3 \%$ (R), 77,3\% (CB) y $63,6 \%$ (W). Comparando la técnica de flotación con las de sedimentación, se observó que $B$. hominis, G. lamblia, A. lumbricoides y $T$. trichiura se recuperaron con mayor frecuencia mediante sedimentación, resultando mejor el método de Ritchie $(\mathrm{p}<0,05)$. Para $H$. nana fue superior la recuperación por flotación, pero esta diferencia no resultó estadísticamente significativa $(\mathrm{p}>$ $0,05)$.

Otros autores ${ }^{13}$ resaltaron las ventajas de la sedimentación en la recuperación de huevos y larvas de helmintos, pero hallaron más favorable la técnica de flotación de Willis para la observación de quistes de protozoos, huevos de $H$. nana y uncinarias. Estos autores enfatizaron la importancia de usar ambas técnicas de concentración para maximizar la efectividad del diagnóstico coproparasitológico. En otro trabajo, la técnica de Willis fue más efectiva en la recuperación de huevos de geohelmintos que la de Ritchie ${ }^{14}$. Por otra parte, otros investigadores, hallaron que la flotación fue más eficiente que la sedimentación en el caso de $C$. parvum $^{15}$, mientras que en USA no se observó diferencias significativas entre estas técnicas en la recuperación de C. parvum $^{16}$. Recientemente, autores cubanos plantearon la 
Eficiencia diagnóstica tres métodos de enriquecimiento coproparasitológico - G. T. Navone et al.

Tabla 1. Frecuencia de recuperación de parásitos intestinales utilizando tres técnicas coproparasitológicas de concentración

\begin{tabular}{lcrrrrrrr}
\hline \multirow{2}{*}{ Especie } & \multicolumn{2}{c}{ Ritchie } & \multicolumn{9}{c}{ Carles-Barthelemy Willis } & \multirow{2}{*}{ Total } & p \\
& $\mathbf{N}^{\circ}$ & $\mathbf{\%}$ & $\mathbf{N}^{\circ}$ & $\boldsymbol{\%}$ & $\mathbf{N}^{\circ}$ & $\mathbf{\%}$ & & \\
\hline A. lumbricoides & 27 & 79,4 & 25 & 73,5 & 19 & 55,8 & 34 & $>0,05$ \\
T. trichiura & 7 & 77,7 & 6 & 66,6 & 3 & 33,3 & 9 & $=0,05$ \\
H. nana & 13 & 72,2 & 13 & 72,2 & 15 & 83,3 & 18 & $>0,05$ \\
B. hominis & 53 & 72,6 & 51 & 69,8 & 34 & 46,5 & 73 & $<0,01$ \\
G. lamblia & 46 & 85,2 & 41 & 75,9 & 35 & 64,8 & 54 & $<0,05$ \\
\hline
\end{tabular}

utilidad de dos métodos coproparasitológicos y su empleo en un ensayo terapéutico antigiardiásico ${ }^{17}$. Demostraron que la técnica de Ritchie fue más efectiva que el examen directo para el diagnóstico de G. lamblia, Entamoeba histolytica, Entamoeba dispar y Cyclospora cayetanensis, y se demostró además mayor sensibilidad del examen seriado sobre el análisis de una sola muestra. Esta comparación fue realizada también cuando se suministraron tinidazol y albendazol para el tratamiento de G. lamblia.

En el presente trabajo las muestras analizadas fueron seriadas y pudo comprobarse la efectividad de las técnicas de concentración y en particular la de Ritchie.

Por otra parte, otros investigadores observaron que la frecuencia de diagnóstico de $B$. hominis fue mayor por examen directo que por Ritchie ${ }^{18,19}$. Ellos consideraron que probablemente este parásito se deforme por la centrifugación y quede en el anillo de residuos que resulta de la aplicación de esta técnica de sedimentación. Sin embargo, la frecuencia de diagnóstico de $B$. hominis observada en el presente trabajo fue mayor utilizando esta técnica que los restantes métodos de concentración.

A partir de los resultados obtenidos, el método de Ritchie resultó más eficaz en la recuperación tanto de protozoos como de helmintos. La emergencia de altas prevalencias parasitarias, hace imprescindible una selección metodológica diagnóstica sensible que reduzca el porcentaje de errores en la identificación de protozoos y helmintos, especialmente de aquellas formas que concentran la mayor cantidad de fallas diagnósticas ${ }^{20-22}$. Esta situación permite recomendar la realización de al menos una técnica de sedimentación $(\mathrm{R})$ y una de flotación y se destaca que a medida que se perfeccionen las técnicas diagnósticas, la frecuencia de hallazgos de

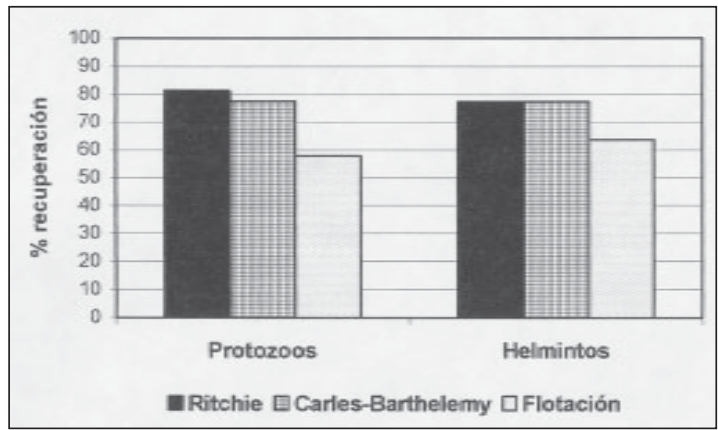

Figura 1. Frecuencia de recuperación de helmintos y protozoos mediante tres técnicas de concentración.

parásitos aumentará, especialmente para aquellos protozoos y helmintos cuyo rol como patógenos resulta emergente de la situación sociocultural de los países en vías de desarrollo. Es importante también proponer acciones tendientes a incorporar métodos avanzados de diagnóstico que puedan promoverse a través de grupos de discusión en talleres y cursos que favorezcan la utilización de técnicas modernas y efectivas.

\section{RESUMEN}

Se analizaron 165 muestras fecales seriadas, utilizando dos métodos de sedimentación: Ritchie (R) y Carles Barthelemy (CB) y uno de flotación: Willis (W), con el fin de optimizar el diagnóstico de los parásitos intestinales y determinar la eficacia de las técnicas. Se hallaron parásitos en $119(72,1 \%)$ de los analizados. Hubo diferencias significativas en la recuperación de protozoos ( $\mathrm{p}$ $<0,001$ ), observándose 81,4\% (R), 77,4\% (CB), y $57,8 \%$ (W). Blastocystis hominis, G.lamblia, Ascaris lumbricoides y Trichuris trichiura se recuperaron con mayor frecuencia mediante sedimentación, resultando más efectivo el método de Ritchie $(\mathrm{p}<0,05)$. 


\section{REFERENCIAS}

1.- SAVIOLI L, BUNDY DAP, TOMKINS A. Intestinal parasitic infections: a soluble public health problem. Trans R Soc Trop Med Hyg 1992; 86: 353-4.

2.- CHAN M S. The global burden of intestinal nematode infections. Fifty years on. Parasitol Today 1997; 113: 438-43.

3.- MARSHALL M M, NAUMOVITZ D, ORTEGA Y, STERLING C R. Waterborne protozoan pathogens. Clin Microbiol Rev 1997; 10: 67-85.

4.- GRIFFITHS J R. Human cryptosporidiosis. Epidemiology, trasmisión, clinical disease, treatment and diagnosis. Adv Parasitol 1998; 40: 38-72.

5.- GAMBOA M I, BASUALDO FARJAT J A, et al. Prevalence of intestinal parasitosis within three population groups in La Plata, Buenos Aires, Argentina. European J Epidemiol 1998; 14: 55-61.

6.- GAMBOA M I, BASUALDO J A, CÓRDOBA M A, et al. Distribution of Intestinal parasitoses in relation to environmental and sociocultural parameters In La Plata, Argentina. J Helminthol 2003; 77: 15-20.

7.- CORDOBA A, CIARMELA L, PEZZANI B, et al. Presencia de parásitos intestinales en paseos públicos urbanos en La Plata, Argentina. Parasitol Latinoam 2002; 57: 25-9.

8.- ORIHEL T C, ASH L R. Medios auxiliares para el diagnóstico de las parasitosis intestinales. Geneva. Organización Mundial de la Salud; 1997.

9.- GARCÍA L S. Macroscopic and microscopic examination of fecal specimens. In Diagnostic medical parasitology. ASM Press, Washington D.C. 2001: 74185.

10.- MENDEZ O C. Lecciones prácticas sobre enteroparasitosis humanas. Edit. Federación Bioquímica de la Provincia de Buenos Aires, Argentina 1998; 165 p.

11- FELDMAN R E, GUARDIS M V. Diagnóstico coproparasitológico. Fundamentos, normas, metodología, bioseguridad, control de calidad. Nueva guía práctica. (Coproparasitological diagnostic. Ground, norms, metodology, biohazard, quality control. New practice guide). Revista de la Federación Bioquímica de la Provincia de Buenos Aires. La Plata. Argentina, 1990, 65 p.
12.- TRUANT A L, ELLIOTT S H, KELLY M T, SMITH $\mathrm{J}$ H. Comparison of formalin-ethyl ether sedimentation, formalin-ethyl acetate sedimentation, and zinc sulfate flotation techniques for detection of intestinal parasites. J Clin Microbiol 1981; 13: 882-4.

13.- ATIAS A, NEGHME A. Parasitología clínica, $3^{a}$ edición. Edit. Mediterráneo, Santiago de Chile, 1996; $618 \mathrm{p}$.

14.- NUNEZ-FERNÁNDEZ F A, SANJURJO GONZÁLEZ E, FINLAY VILLALVILLA C M. Comparison of several coproparasitological techniques for the diagnosis of soil-transmitted intestinal helminthiasis. Rev Inst Med Trop Sao Paulo 1991; 33: 403-6.

15.- DENG M Q, CLIVER D O. Comparative detection of Cryptosporidium parvum oocysts from apple juice. Int J Food Microbiol 2000; 54: 155-62.

16.- MC NABB S J, HENSEL D M, WELCH DF, et al. Comparison of sedimentation and flotation techniques for identification of Cryptosporidium sp. oocysts in a large outbreak of human diarrhea. J Clin Microbiol 1985; 22: 587-9.

17.- MENDOZA D, NUÑEZ F A, ESCOBEDO A A, et al. Utilidad de dos métodos coproparasitológicos y su empleo en un ensayo terapéutico antigiardiásico. Rev Cubana Med Trop 2003; 55: 174-8.

18.- WANG L C. Improvement in the identification of intestinal of intestinal parasites by a ConcentratedMerthiolate-Iodine-Formaldehyde Technique. J Parasitol 1998; 84: 457-8.

19.- PARIJA S C, SRINIVASA H. Viewpoint: The neglect of stool microscopy for intestinal parasites and possible solutions. Trop Med Intern Health 1999; 522-4.

20.- LAIRD R, RAMÍREZ E, DEL RISCO U, et al. Intervención para mejorar el diagnóstico coproparasitológico en la provincia "Las Tunas", Cuba, 19931994. Bol Chil Parasitol 51: 97-100.

21.- NÚÑEZ F A, GINORIO D, FINLAY C M. Control de calidad del diagnóstico coproparasitológico en la Provincia de Ciudad Habana, Cuba. Cad Saude Publica, 1997; 13: 67-72.

22.- NÚNEEZ FA, GINORIO D, CORDOVI R, FINLAY C M. Intervención educativa para mejorar la calidad del el diagnóstico coproparasitológico en la red de salud de Ciudad Habana, Cuba. Cad Saude Publica 1998; 14: 13944. 